\title{
SYMMETRIC LIE MODELS OF A TRIANGLE
}

\author{
URTZI BUIJS, YVES FÉLIX, ANICETO MURILLO, AND DANIEL TANRÉ
}

\begin{abstract}
R. Lawrence and D. Sullivan have constructed a Lie model for an interval from the geometrical idea of flat connections and flows of gauge transformations. Their model supports an action of the symmetric group $\Sigma_{2}$ reflecting the geometrical symmetry of the interval. In this work, we present a Lie model of the triangle with an action of the symmetric group $\Sigma_{3}$ compatible with the geometrical symmetries of the triangle. We also prove that the model of a graph consisting of a circuit with $k$ vertices admits a Maurer-Cartan element stable by the automorphisms of the graph.
\end{abstract}

\section{INTRODUCTION}

In rational homotopy theory, the existence of algebraic models of topological spaces in terms of Lie algebras was first established by D. Quillen in [8] for simply connected spaces. His work is based on a succession of couples of adjoint functors between the category of simply connected spaces and the category of differential Lie algebras, positively graded. The complexity of the Quillen functors contrasts with the simple couple of functors $\left(A_{P L},\langle-\rangle\right)$ introduced by D. Sullivan ([10]) between the category of simplicial sets and the category of commutative differential graded algebras (cdga in short). Sullivan's construction is based on a contractible simplicial cdga $\Omega$ • defined by

$$
\Omega_{n}=\mathbb{Q}\left[t_{0}, \ldots, t_{n}\right] \otimes \wedge\left(d t_{0}, \ldots, d t_{n}\right) /\left(\sum t_{i}-1, \sum d t_{i}\right),
$$

with the $t_{i}$ 's in degree zero and the $d t_{i}$ 's in degree one. The cdga $A_{P L}(X)$ associated to a simplicial set $X$ is defined from the set of simplicial maps from $\Omega_{\bullet}$ to $X$.

A natural question is the existence of a similar construction in the context of Lie algebras. We solve it in [1] from the cocommutative, coassociative, infinity coalgebra structure on the rational singular chains and a transfer process. For a finite simplicial set, $K$, this gives a complete differential graded Lie algebra $\mathfrak{L}(K)$ which contains information on the rational homotopy type of $K$, as the Bousfield-Kan completion of its fundamental group for instance. The departure point is the construction of a family $\mathfrak{L}_{k}=\mathfrak{L}\left(\Delta^{k}\right)$ which extends the construction by R. Lawrence and D. Sullivan of a complete differential Lie algebra $\mathfrak{L}_{1}$ in the case of the interval, see [6]. We recall it in the first section. Their model is symmetric for the action of the symmetric group $\Sigma_{2}$ deduced from the geometry of the interval.

Date: July 31, 2018.

2010 Mathematics Subject Classification. 55P62, 17B01, 55U10.

Key words and phrases. Rational homotopy theory. Lie models of simplicial sets. Symmetric models.

The first and third authors have been partially supported by the Junta de Andalucía grant FQM-213. The fourth author has been partially supported by the ANR-11-LABX-0007-01 "CEMPI". The authors have been partially supported by the MINECO grants MTM2013-41768-P and MTM2016-78647-P. 
In Section 3, we recall the construction of a Lie model $\mathfrak{L}_{2}$ of the triangle $\Delta^{2}$. Based on a vertex of $\Delta^{2}$, this model does not reflect the symmetries of a triangle. We modify it in a Lie model with an action of the symmetric group $\Sigma_{3}$ compatible with the different symmetries. For that, we consider the geometrical action of $\Sigma_{3}$ on the generators of $\mathfrak{L}_{2}$ and prove the existence of a Maurer-Cartan element stable by any element of $\Sigma_{3}$. Even though the differential in $\mathfrak{L}_{2}$ is, up to some extend, uniquely determined (Proposition 3.1), there are many Maurer-Cartan elements invariant by $\Sigma_{3}$.

The Maurer-Cartan elements play the role of points and are recalled in Section 1. Therefore, a Maurer-Cartan element invariant by the action of $\Sigma_{3}$ is an algebraic representation of the barycentre of the triangle. After the interval of Lawrence and Sullivan, this is the second stage in the research of a Lie $\Sigma_{n}$-model of a simplex $\Delta^{n}$.

Our first step consists to prove that the Lie model $\mathfrak{L}(\Gamma)$, of a graph $\Gamma$ consisting of a circuit with $k$ vertices, contains a Maurer-Cartan element invariant by the automorphisms of the graph.

In all this work, vector spaces and chain complexes are $\mathbb{Z}$-graded and we denote by $|x|$ the degree of an element. The free Lie algebra on a rational vector space $V$ is represented by $\mathbb{L}(V)$. The vector subspace $\mathbb{L}^{i}(V)$ is the set of elements of $\mathbb{L}(V)$ of bracket length $i$. The objects considered in this work are complete free Lie algebras, defined by

$$
\widehat{\mathbb{L}}(V)={\underset{\iota}{i}}_{\lim } \mathbb{L}(V) / \mathbb{L} \geq^{i}(V) .
$$

Endowed with a Lie differential, they are called complete differential graded Lie algebras (henceforth cdgl) and, if the explicit elicitation of the vector space $V$ is not necessary, we denote also $L=\widehat{\mathbb{L}}(V)$. If $x \in L$, the $\operatorname{map} \operatorname{ad}_{x}: L \rightarrow L$ is the adjoint derivation $y \mapsto[x, y]$.

\section{BACKGROUND}

The Baker-Campbell-Hausdorff product (henceforth BCH-product) of two elements $x$ and $y$ of degree 0 in a free complete Lie algebra $\widehat{\mathbb{L}}(V)$ is defined by

$$
x * y=\log \left(e^{x} e^{y}\right) .
$$

This product satisfies the following properties (see [1], [5] or [6] for instance) for any $x$, $y, z$ in $L_{0}$ and $r, s$ in $\mathbb{Q}$ :

(i) $x *(y * z)=(x * y) * z$,

(ii) $(r x) *(s x)=(r+s) x$. In particular, the inverse of $x$ is $-x$; i.e., $(x) *(-x)=0$,

(iii) $-(y * x)=(-x) *(-y)$,

(iv) $\operatorname{ad}_{x * y}=\operatorname{ad}_{x} * \operatorname{ad}_{y}$ and $e^{\operatorname{ad}_{x * y}}=e^{\operatorname{ad}_{x}} \circ e^{\operatorname{ad}_{y}}$,

(v) $e^{\operatorname{ad}_{x}}(y)=x * y *(-x)$ and $e^{\operatorname{ad}_{x}}(y) * e^{\operatorname{ad}_{x}}(z)=e^{\operatorname{ad}_{x}}(y * z)$,

(vi) $r(x * y) * x=x * r(y * x)$ and $r(y * x) *(-x)=(-x) * r(x * y)$.

As the $\mathrm{BCH}$-product is associative, we can introduce the following notation, for any family $\left(w_{i}\right)_{1 \leq i \leq r}$ of elements in $L_{0}$,

$$
*_{i=1}^{r} w_{i}=w_{1} * w_{2} * \cdots * w_{r} .
$$

The next property follows from the Dynkin formula for the BCH-product, see [9]. 
Lemma 1.1. Let $\left(w_{i}\right)_{1 \leq i \leq r}$ be elements of degree zero in $\widehat{\mathbb{L}}(V)$ that coincide in the quotient $\widehat{\mathbb{L}}(V) / \widehat{\mathbb{L}}^{>n}(V)$. Then, for any rational numbers $\lambda_{i}$, we have an equality between the classes modulo $\widehat{\mathbb{L}}^{>n+1}(V)$,

$$
\left[*_{i=1}^{r} \lambda_{i} w_{i}\right]^{<n+1}=\left[\sum_{i=1}^{r} \lambda_{i} w_{i}\right]^{<n+1} .
$$

Given a $\operatorname{cdgl}(L, d)$, a Maurer-Cartan element (in short a MC-element) is an element $a \in L_{-1}$ such that

$$
d a+\frac{1}{2}[a, a]=0
$$

If $a$ is a MC-element in a cdgl $(L, d)$, the derivation defined by

$$
d_{a} x=\operatorname{ad}_{a}(x)+d x, x \in L,
$$

is a differential.

Denote by $\operatorname{MC}(L)$ the set of MC-elements of $L$. There is an action of the complete Lie algebra of elements of degree $0, L_{0}$, on $\mathrm{MC}(L)$, called gauge action, and defined as follows:

$$
x \mathcal{G} a=e^{\operatorname{ad}_{x}}(a)-\frac{e^{\operatorname{ad}_{x}}-1}{\operatorname{ad}_{x}}(d x)=\sum_{i \geq 0} \frac{\operatorname{ad}_{x}^{i}(a)}{i !}-\sum_{i \geq 0} \frac{\operatorname{ad}_{x}^{i}(d x)}{(i+1) !},
$$

with $x \in L_{0}, a \in \operatorname{MC}(L)$, see [2, Proposition 1.2]. If $L=\widehat{\mathbb{L}}(V)$, the linear and the quadratic parts of the gauge action can be determined explicitly as,

$$
\begin{aligned}
(x \mathcal{G} a)_{1} & =a_{1}-(d x)_{1}, \\
(x \mathcal{G} a)_{2} & =a_{2}+\left[x_{1}, a_{1}\right]-\frac{1}{2}\left[x_{1}, d_{1} x_{1}\right]-d_{2} x_{1}-d_{1} x_{2},
\end{aligned}
$$

where the decompositions along the bracket length are denoted $a=\sum_{i \geq 1} a_{i}, x=\sum_{i \geq 1} x_{i}$, $d=\sum_{i \geq 1} d_{i}$ with $d_{i}(V) \subset \mathbb{L}^{i}(V)$.

The gauge action is compatible with the $\mathrm{BCH}$-product in $L_{0}$ : for any $x, y \in L_{0}$ and $a \in \mathrm{MC}(L)$, we have

$$
x \mathcal{G} y \mathcal{G} a=(x * y) \mathcal{G} a .
$$

(In the context of model of the interval, this property is a consequence of [6, Theorem 2] but it also appears in different works, see [4, Appendix B] for instance.) We denote by $\widetilde{\mathrm{MC}}(L)$ the set of equivalence classes for the gauge action.

In [6], R. Lawrence and D. Sullivan have defined a Lie model $\mathfrak{L}_{1}$ of the interval $\Delta^{1}$ :

$$
\mathfrak{L}_{1}=(\widehat{\mathbb{L}}(a, b, x), d)
$$

with $|a|=|b|=-1,|x|=0, d a=-\frac{1}{2}[a, a], d b=-\frac{1}{2}[b, b]$ and

$$
\begin{aligned}
d x & =\operatorname{ad}_{x} a+\frac{\operatorname{ad}_{-x}}{e^{-\operatorname{ad}_{x}-1}}(b-a)=\frac{\operatorname{ad}_{x}}{1-e^{\operatorname{ad}_{x}}} a+\frac{\operatorname{ad}_{x}}{1-e^{-\mathrm{ad}_{x}}} b \\
& =\operatorname{ad}_{x} b+\frac{\operatorname{ad}_{x}}{e^{\operatorname{ad}_{x}}-1}(b-a) .
\end{aligned}
$$


The cdgl $\mathfrak{L}_{1}$ is called the LS-interval. The elements $a$ and $b$ are MC-elements. The set of MC-elements of $\mathfrak{L}_{1}$ has been characterized in [2] and the unicity of such model is established in [7]. As it was observed by Lawrence and Sullivan, an element $x$ of degree 0 in a $\operatorname{cdgl}(\widehat{\mathbb{L}}(V), d)$ defines a flow by

$$
\frac{d v}{d t}=d x-\operatorname{ad}_{x}(v),|v|=-1
$$

The flowing by $x$ from $a$ to $b$, in time 1 , corresponds to

$$
(-x) \mathcal{G} a=b
$$

which determines the expression (6) of the differential of $x$. Such $x$ is called a path from $a$ to $b$. The differential $d x$ is clearly stable by the symmetry $(\tau: a \leftrightarrow b, \tau x=-x)$ which generates the symmetric group $\Sigma_{2}$. Mention also the existence of a symmetric model of a 2-gon in [5], where the geometrical action of $\Sigma_{2}$ is reflected on the differential of its model.

The next proposition recalls the well known relation between $d_{a}$ and $d_{b}$ in $\mathfrak{L}_{1}$. We give the proof for completeness.

Proposition 1.2. Let $(L, d)$ be a cdgl containing a LS-interval $(\widehat{\mathbb{L}}(a, b, x), d)$. Then, for any $w \in L$, we have

$$
d_{a} e^{\operatorname{ad}_{x}}(w)=e^{\operatorname{ad}_{x}}\left(d_{b} w\right) .
$$

Proof. From [6, Lemma 1], we have

$$
d_{b}\left(e^{-\operatorname{ad}_{x}} w\right)=e^{-\operatorname{ad}_{x}}\left(d_{b} w\right)+(-1)^{|w|} e^{-\operatorname{ad}_{x}} \operatorname{ad}_{w} \frac{e^{-\operatorname{ad}_{x}}-1}{\operatorname{ad}_{x}}\left(d_{b} x\right) .
$$

Replacing $d_{b} x$ by its value (7) gives

$$
\begin{aligned}
d_{b}\left(e^{\left.-\operatorname{ad}_{x} w\right)}\right. & =e^{-\operatorname{ad}_{x}}\left(d_{b} w+(-1)^{|w|} \operatorname{ad}_{w}(b-a)\right) \\
& =e^{-\operatorname{ad}_{x}}\left(d_{a} w\right) .
\end{aligned}
$$

\section{LIE MODEL OF A GRAPH CONSISTING OF A CIRCUIT}

In this section we consider cdgl's endowed with the action of a group $G$.

Definition 2.1. A $G$-cdgl is a $\operatorname{cdgl}(L, d)$, with a group action $G \times L \rightarrow L$, compatible with the differential and the Lie bracket; i.e., for any $v, w \in L$ and $\varphi \in G$, we have

$$
\varphi[v, w]=[\varphi(v), \varphi(w)] \text { and } \varphi(d v)=d(\varphi(v)) .
$$

Let $\Gamma$ be a graph consisting of a circuit with $k$ vertices. Then $\Gamma$ is the union of $k$ intervals and its Lie model is a concatenation of LS-intervals ([6]); i.e.,

$$
\mathfrak{L}(\Gamma)=\left(\widehat{\mathbb{L}}\left(\oplus_{i=1}^{k}\left(\mathbb{Q} \mathrm{v}_{i} \oplus \mathbb{Q} \mathrm{x}_{i}\right), d\right),\right.
$$

where the $\mathrm{v}_{i}$ are MC-elements and the sub dgl's $\left(\widehat{\mathbb{L}}\left(\mathrm{v}_{i}, \mathrm{v}_{i+1}, \mathrm{x}_{i}\right), d\right)$ are LS-intervals. Here by convention, we set $\mathrm{v}_{k+1}=\mathrm{v}_{1}, \mathrm{x}_{k+1}=\mathrm{x}_{1}$. 
The group $G$ of automorphisms of $\Gamma$ is the dihedral group $D_{2 k}$ generated by the cyclic permutation $\sigma$ and the involution $\tau$ on the first vertex,

$$
G=<\sigma, \tau ; \sigma^{k}, \tau^{2}, \sigma \tau=\tau \sigma^{-1}>.
$$

The $\operatorname{cdgl} \mathfrak{L}(\Gamma)$ is a $G$-cdgl. The actions of $\sigma$ and $\tau$ are defined by:

$$
\sigma\left(\mathrm{x}_{i}\right)=\mathrm{x}_{i+1}, \quad \sigma\left(\mathrm{v}_{i}\right)=\mathrm{v}_{i+1}, \quad \tau\left(\mathrm{v}_{i}\right)=\mathrm{v}_{k-i+2}, \quad \tau\left(\mathrm{x}_{i}\right)=-\mathrm{x}_{k-i+1} .
$$

Denote by $V$ the graded vector space generated by the $\mathrm{x}_{i}$ and the $\mathrm{v}_{i}$. We decompose the differential $d$ in $\mathfrak{L}(\Gamma)$ in the form $d=\sum_{i \geq 1} d_{i}$ with $d_{i}(V) \subset \mathbb{L}^{i}(V)$.

Lemma 2.2. With the above notation, the following properties are verified.

(i) Any $d_{1}$-cycle of degree 0 in $\widehat{\mathbb{L}} \geq 2(V)$ is equal to zero.

(ii) An element $w$ of degree zero is such that $w \mathcal{G} \mathrm{v}_{1}=\mathrm{v}_{1}$ if and only if $w=\lambda\left(\mathrm{x}_{1} *\right.$ $\mathrm{x}_{2} * \cdots * \mathrm{x}_{k}$ ) for some rational number $\lambda$.

Proof. (i) Since $H\left(\widehat{\mathbb{L}}(V), d_{1}\right)=\widehat{\mathbb{L}}\left(H\left(V, d_{1}\right)\right)$, we are reduced to the determination of the homology of the 1-dimensional simplicial complex defined by the graph $\Gamma$. We obtain,

$$
H\left(\widehat{\mathbb{L}}(V), d_{1}\right)=\widehat{\mathbb{L}}\left(\mathrm{v}_{1}, \mathrm{x}_{1}+\mathrm{x}_{2}+\cdots+\mathrm{x}_{k}\right),
$$

and any cycle of degree 0 having a zero linear part is a boundary. Since $\widehat{\mathbb{L}}(V)$ has no element of strictly positive degree, this cycle must be 0 .

(ii) First, from (5), we have $\lambda\left(\mathrm{x}_{1} * \cdots * \mathrm{x}_{k}\right) \mathcal{G v}_{1}=\mathrm{v}_{1}$. Now, let $w$ such that $w \mathcal{G v}_{1}=\mathrm{v}_{1}$. Using (3), we get $\mathrm{v}_{1}-d_{1} w_{1}=\mathrm{v}_{1}$ and $d_{1} w_{1}=0$. This implies the existence of $\lambda \in \mathbb{Q}$ such that $w_{1}=\lambda\left(\sum_{i=1}^{k} \mathrm{x}_{i}\right)$. The element $z=\left(-\lambda\left(\mathrm{x}_{1} * \cdots * \mathrm{x}_{k}\right)\right) * w$ verifies $z \mathcal{G} \mathrm{v}_{1}=\mathrm{v}_{1}$ and has a zero linear part. From (4), we deduce that its quadratic part verifies $d_{1} z_{2}=0$. From (i), this implies $z_{2}=0$. By induction, we have $z_{i}=0$ for all $i$ and $w=\lambda\left(\mathrm{x}_{1} * \cdots * \mathrm{x}_{k}\right)$.

Lemma 2.3. With the previous notation, any $w \in \widehat{\mathbb{L}}\left(\oplus_{i=1}^{k} \mathbb{Q} \mathbf{x}_{i}\right)$ such that $\sigma\left(d_{1} w\right)=d_{1} w$ is invariant by $\sigma$; i.e., $\sigma(w)=w$.

Proof. Since $\sigma$ preserves the bracket length, we can suppose that $w \in \mathbb{L}^{\ell}\left(\oplus_{i=1}^{k} \mathbb{Q} \mathbf{x}_{i}\right)$ for some $\ell \geq 1$. In the enveloping algebra, $T\left(\oplus_{i=1}^{k} \mathbb{Q} \mathrm{x}_{i}\right), w$ can be written in an unique way as

$$
w=\sum_{i=1}^{k} \mathrm{x}_{i} X_{i} \text { with } X_{i} \in T^{\ell-1}\left(\oplus_{i=1}^{k} \mathbb{Q} \mathbf{x}_{i}\right) .
$$

Denoting $\nu_{i}=d_{1} \mathrm{x}_{i}=\mathrm{v}_{i+1}-\mathrm{v}_{i}$, we have $\sum_{i=1}^{k} \nu_{i}=0$ and

$$
\begin{aligned}
d_{1} w & =\sum_{i=1}^{k-1} \nu_{i} X_{i}-\left(\sum_{i=1}^{k-1} \nu_{i}\right) X_{k}+\sum_{i=1}^{k} \mathrm{x}_{i} d_{1} X_{i}=\sum_{i=1}^{k-1} \nu_{i}\left(X_{i}-X_{k}\right)+\sum_{i=1}^{k} \mathrm{x}_{i} d_{1} X_{i}, \\
\sigma\left(d_{1} w\right) & =\sum_{i=1}^{k-1} \nu_{i}\left(\sigma X_{i-1}-\sigma X_{k-1}\right)+\sum_{i=1}^{k} \mathrm{x}_{i+1} \sigma\left(d_{1} X_{i}\right) .
\end{aligned}
$$

The hypothesis $\sigma\left(d_{1} w\right)=d_{1} w$ implies

$$
X_{i}-X_{k}=\sigma X_{i-1}-\sigma X_{k-1} .
$$


Thus, the element $\sigma X_{i-1}-X_{i}$ does not depend on $i$. Denoting by $E=\sigma X_{i-1}-X_{i}$ this common value, we have $\sigma X_{i}=X_{i+1}+E$ which implies

$$
\sigma(w)=\sum_{i=1}^{k} \mathrm{x}_{i+1} \sigma X_{i}=\sum_{i=1}^{k} \mathrm{x}_{i+1} X_{i+1}+\left(\sum_{i=1}^{k} \mathrm{x}_{i+1}\right) E=w+\left(\sum_{i=1}^{k} \mathrm{x}_{i}\right) E .
$$

Since $w$ and $\sigma(w)$ are Lie elements, the vector $\left(\sum_{i=1}^{k} \mathbf{x}_{i}\right) E$ must be primitive. Therefore, we have $E=0$ and $\sigma(w)=w$.

Theorem 2.4. The cdgl $\mathfrak{L}(\Gamma)$ contains a MC-element invariant by the actions of $\sigma$ and $\tau$.

Proof. The first step of the proof consists in a construction by induction of a MC-element stable by the action of $\sigma$. We define

$$
P[1]=-\left(\sum_{i=1}^{k-1} \frac{k-i}{k} \mathbf{x}_{i}\right) \mathcal{G} \mathrm{v}_{1} .
$$

Using the property (3) of the gauge action, one has the linear part $P[1]_{1}$ of $P[1]$ :

$$
P[1]_{1}=\mathrm{v}_{1}+\sum_{i=1}^{k-1} \frac{k-i}{k} d_{1} \mathrm{x}_{i}=\frac{1}{k} \sum_{i=1}^{k} \mathrm{v}_{i} .
$$

Therefore, $P[1]$ is a MC-element whose linear part is $\sigma$-invariant. By induction we suppose to have constructed the MC-elements, $P[i]$, for $i=1, \ldots, n-1$, such that

(a) $P[i]-P[i-1] \in \mathbb{L}^{\geq i}(V)$,

(b) $\sigma P[i]_{j}=P[i]_{j}$, for any $j \leq i$, where $P[i]_{j}$ denotes the component of $P[i]$ in the Lie brackets of length $j$,

As the graph $\Gamma$ is connected, from [3, Theorem] there is only one non-trivial equivalence class of MC-elements. Thus, there is an element of degree $0, T$, satisfying the equation

$$
T \mathcal{G} P[n-1]=\sigma P[n-1] .
$$

Formula (3) gives the following equality between the linear parts,

$$
P[n-1]_{1}-d_{1} T_{1}=\sigma P[n-1]_{1} .
$$

Since $P[n-1]_{1}=\sigma P[n-1]_{1}, d_{1} T_{1}=0$ and $T_{1}$ is $\sigma$-invariant. We prove by induction on $j$ that $T_{j}$ is $\sigma$-invariant for $j<n$. We thus suppose $T_{1}, \ldots, T_{r-1}$ to be $\sigma$-invariant. Then from $T \mathcal{G P}[n-1]=\sigma P[n-1]$, we get that $d_{1} T_{r}$ is $\sigma$-invariant, and by Lemma 2.3, $T_{r}$ is $\sigma$-invariant.

The same construction in Lie brackets of length $n$ gives a $\sigma$-invariant element, $S$, such that

$$
P[n-1]_{n}+S-d_{1} T_{n}=\sigma P[n-1]_{n} .
$$

With successive applications of $\sigma$, we deduce the system of equations,

$$
\begin{aligned}
P[n-1]_{n}+S-d_{1} T_{n} & =\sigma P[n-1]_{n}, \\
\sigma P[n-1]_{n}+S-d_{1} \sigma T_{n} & =\sigma^{2} P[n-1]_{n}, \\
\cdots & =\ldots, \\
\sigma^{k-1} P[n-1]_{n}+S-d_{1} \sigma^{k-1} T_{n} & =P[n-1]_{n} .
\end{aligned}
$$


The adding of these equations gives

$$
k S=d_{1}\left(T_{n}+\sigma T_{n}+\cdots+\sigma^{k-1} T_{n}\right) .
$$

We define $T^{\prime}$ and $P^{\prime}$ by

$$
T_{i}^{\prime}= \begin{cases}T_{i}, & \text { for } i<n \\ \frac{k-1}{k} T_{n}+\frac{k-2}{k} \sigma T_{n}+\cdots+\frac{1}{k} \sigma^{k-2} T_{n} & \text { for } i=n, \\ 0 & \text { for } i>n\end{cases}
$$

and

$$
P^{\prime}=T^{\prime} \mathcal{G}[n-1] .
$$

By construction, $P_{<n}^{\prime}=P[n-1]_{<n}$. On the other hand,

$$
P_{n}^{\prime}=P[n-1]_{n}+S^{\prime}-\sum_{i=0}^{k-2} \frac{k-i-1}{k} d_{1} \sigma^{i} T_{n},
$$

with $S^{\prime}$ invariant by $\sigma$. We apply $\sigma$ to this equality and obtain:

$$
\sigma P_{n}^{\prime}=\sigma P[n-1]_{n}+S^{\prime}-\sum_{i=0}^{k-2} \frac{k-i-1}{k} d_{1} \sigma^{i+1} T_{n} .
$$

Using (11) and extracting the value of $\sigma^{k-1} T_{n}$ of (12), we have

$$
\begin{gathered}
\sigma P_{n}^{\prime}=P[n-1]_{n}+S-d_{1} T_{n}+S^{\prime}-\sum_{i=0}^{k-3} \frac{k-i-1}{k} d_{1} \sigma^{i+1} T_{n} \\
-\frac{1}{k}\left(k S-\sum_{i=0}^{k-2} d_{1} \sigma^{i} T_{n}\right) .
\end{gathered}
$$

With a reordering of the indices, this formula becomes

$$
\begin{aligned}
\sigma P_{n}^{\prime} & =P[n-1]_{n}+S^{\prime}-\sum_{j=0}^{k-2} \frac{k-j-1}{k} d_{1} \sigma^{j} T_{n} \\
& =P_{n}^{\prime} .
\end{aligned}
$$

We write $P[n]=P^{\prime}$ and the inductive step is closed. Now by property (a) of the induction, the sequence $P[n]$ converges to a MC-element $P$ with $\sigma P=P$.

We now use $P$ to construct a MC-element $\Omega$ invariant by $\sigma$ and $\tau$. Since $\sigma \tau=\tau \sigma^{k-1}$, we deduce that $\tau P$ is also $\sigma$-invariant. On the other hand, since $P$ and $\mathrm{v}_{1}$ are gauge equivalent, there is an element $\mathrm{w}$ such that $\mathrm{w} \mathcal{G} P=\mathrm{v}_{1}$ and we set

$$
\alpha=(-\tau \mathrm{w}) * \mathrm{w} ;
$$

i.e., $\alpha$ can be represented as a composition of paths,

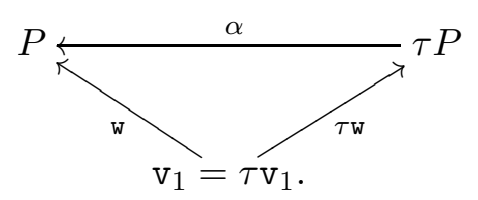


From (8) and from Property (iii) of the BCH-product, recalled in Section 1, we get

$$
\begin{aligned}
\alpha \mathcal{G P} & =\tau P, \\
\tau \alpha & =(-\mathrm{w}) * \tau \mathrm{w}=-((-\tau \mathrm{w}) * \mathrm{w})=-\alpha .
\end{aligned}
$$

We define a MC-element $\Omega$ by

$$
\Omega=\left(\frac{\alpha}{2}\right) \mathcal{G P}
$$

and deduce that

$$
\tau \Omega=\left(-\frac{\alpha}{2}\right) \mathcal{G} \tau P=\frac{\alpha}{2} \mathcal{G}(-\alpha) \mathcal{G} \tau P=\frac{\alpha}{2} \mathcal{G} P=\Omega .
$$

Thus the MC-element $\Omega$ is $\tau$-invariant. We prove now that $\alpha$ is $\sigma$-invariant which implies the $\sigma$-invariance of $\Omega$.

The linear part of $\alpha \mathcal{G P}=\tau P$ is $P_{1}-d_{1} \alpha_{1}=\tau P_{1}$. This gives the $\sigma$-invariance of $d_{1} \alpha_{1}$ and by Lemma 2.2(i) the $\sigma$-invariance of $\alpha_{1}$. Suppose that the components $\alpha_{j}$ of $\alpha$, in the Lie brackets of length $j$, are $\sigma$-invariant for any $j \leq n$. The component of the equality $\alpha \mathcal{G} P=\tau P$ in the Lie bracket of length $(n+1)$ gives

$$
P_{n+1}+S-d_{1} \alpha_{n+1}=(\tau P)_{n+1},
$$

where $S$ is $\sigma$-invariant by induction. This implies the $\sigma$-invariance of $d_{1} \alpha_{n+1}$ and therefore of $\alpha_{n+1}$ as required.

\section{Symmetric Lie model of a triangle}

Let $\Delta^{2}$ be the simplicial complex of the triangle, with 3 vertices and 3 edges. In the spirit of the model of the interval ([6]), we are looking for a model of the triangle as a cdgl whose generators are the simplices of $\Delta^{2}$, whose linear part of the differential is the differential of the associated simplicial complex and whose the sub-cdgl's corresponding to edges are LS-intervals. We call it a Lie model of $\Delta^{2}$. The next result proves existence and unicity of such model.

Proposition 3.1. Let $\Delta^{2}$ be the simplicial complex of the triangle.

1) A Lie model of $\Delta^{2}$ is the cdgl

$$
\mathfrak{L}_{2}=\left(\widehat{\mathbb{L}}\left(\mathrm{v}_{1}, \mathrm{v}_{2}, \mathrm{v}_{3}, \mathrm{x}_{1}, \mathrm{x}_{2}, \mathrm{x}_{3}, \mathrm{e}\right), d\right),
$$

with $\left|\mathrm{v}_{i}\right|=-1,\left|\mathrm{x}_{i}\right|=0$ and $|\mathrm{e}|=1$. The elements $\mathrm{v}_{i}$ are MC-elements, the intervals $\left[\mathrm{v}_{1}, \mathrm{v}_{2}, \mathrm{x}_{1}\right],\left[\mathrm{v}_{2}, \mathrm{v}_{3}, \mathrm{x}_{2}\right]$ and $\left[\mathrm{v}_{3}, \mathrm{v}_{1}, \mathrm{x}_{3}\right]$ are LS-intervals, and

$$
d \mathrm{e}=\left[\mathrm{e}, \mathrm{v}_{1}\right]+\mathrm{x}_{1} * \mathrm{x}_{2} * \mathrm{x}_{3} .
$$

2) Let $\mathfrak{L}_{2}$ be as before and $\left(\widehat{\mathbb{L}}\left(\mathrm{v}_{1}, \mathrm{v}_{2}, \mathrm{v}_{3}, \mathrm{x}_{1}, \mathrm{x}_{2}, \mathrm{x}_{3}, \mathrm{e}\right), d^{\prime}\right)$ be a cdgl such that

(i) the differentials $d$ and $d^{\prime}$ coincide on the generators $\mathrm{v}_{i}$ and $\mathrm{x}_{i}$,

(ii) the linear parts $d_{1} \mathrm{e}$ and $d_{1}^{\prime} \mathrm{e}$ coincide,

(iii) $d_{\mathrm{v}_{1}}^{\prime}(\mathrm{e}) \in \widehat{\mathbb{L}}\left(\mathrm{x}_{1}, \mathrm{x}_{2}, \mathrm{x}_{3}\right)$.

Then, we have $d=d^{\prime}$. 
Proof. 1) The proof is reduced to: " $\mathrm{x}_{1} * \mathrm{x}_{2} * \mathrm{x}_{3}$ is a $d_{\mathrm{v}_{1}}$-cycle." Let $\mathfrak{L}_{1}=(\widehat{\mathbb{L}}(a, b, x), d)$ be a LS-interval. The composition of morphisms as in [6, Theorem 2] gives a cdgl map

$$
\varphi: \mathfrak{L}_{1} \rightarrow\left(\widehat{\mathbb{L}}\left(\mathrm{v}_{1}, \mathrm{v}_{2}, \mathrm{v}_{3}, \mathrm{x}_{1}, \mathrm{x}_{2}, \mathrm{x}_{3}\right), d\right),
$$

with $\varphi(a)=\varphi(b)=\mathrm{v}_{1}$ and $\varphi(x)=\mathrm{x}_{1} * \mathrm{x}_{2} * \mathrm{x}_{3}$. We deduce

$$
\begin{aligned}
d\left(\mathrm{x}_{1} * \mathrm{x}_{2} * \mathrm{x}_{3}\right) & =d \varphi(x)=\varphi(d x) \\
& =\varphi\left(\operatorname{ad}_{x} b+\frac{\operatorname{ad}_{x}}{e^{\operatorname{ad}_{x}-1}}(b-a)\right) \\
& =\varphi[x, b]=-\operatorname{ad}_{\mathrm{v}_{1}}\left(\mathrm{x}_{1} * \mathrm{x}_{2} * \mathrm{x}_{3}\right) .
\end{aligned}
$$

This implies $d_{\mathrm{v}_{1}}\left(\mathrm{x}_{1} * \mathrm{x}_{2} * \mathrm{x}_{3}\right)=0$ as expected.

2) We decompose $d_{\mathrm{v}_{1}}$ and $d_{\mathrm{v}_{1}}^{\prime}$ along the bracket size as $d_{\mathrm{v}_{1}}=\sum_{j \geq 1} d_{j}$ and $d_{\mathrm{v}_{1}}^{\prime}=$ $\sum_{j \geq 1} d_{j}^{\prime}$. By hypothesis, we have $d_{1}=d_{1}^{\prime}$. Suppose, by induction, that $d_{j}=d_{j}^{\prime}$ for any $j<n$. From $d^{2}=d^{\prime 2}=0$, we deduce

$$
d_{1} d_{n}^{\prime}=-\sum_{j=2}^{n} d_{j} d_{n+1-j}^{\prime}=-\sum_{j=2}^{n} d_{j} d_{n+1-j}=d_{1} d_{n} .
$$

Therefore, the element $d_{n} \mathrm{e}-d_{n}^{\prime} \mathrm{e}$ is a $d_{1}$-cycle of degree 0 in $\widehat{\mathbb{L}}\left(\mathrm{x}_{1}, \mathrm{x}_{2}, \mathrm{x}_{3}\right)$. From the first assertion of Lemma 2.2, we deduce $d_{n} \mathrm{e}=d_{n}^{\prime} \mathrm{e}$.

Denote by $\Gamma$ the boundary of the triangle and by $\mathfrak{L}(\Gamma)$ its Lie model. As the symmetric group $\Sigma_{3}$ coincides with the dihedral group $D_{6}$, we may consider the action of $\Sigma_{3}$ on $\mathfrak{L}(\Gamma)$ described at the beginning of Section 2 and apply Theorem 2.4.

Theorem 3.2. Let $\Omega$ be a $\Sigma_{3}$-invariant MC-element in $\mathfrak{L}(\Gamma)$. Then the cdgl $\mathfrak{L}_{2}$ is a $\Sigma_{3}$-cdgl isomorphic to the cdgl $\left(\mathfrak{L}(\Gamma) \widehat{\amalg} \mathbb{L}\left(\mathrm{e}^{\prime}\right), d\right)$ satisfying the following properties:

(i) for some $\beta$ of degree 0 , we have

$$
d \mathrm{e}^{\prime}=-\left[\Omega, \mathrm{e}^{\prime}\right]+\beta * \mathrm{x}_{1} * \mathrm{x}_{2} * \mathrm{x}_{3} *(-\beta)
$$

(ii) $\sigma\left(\mathrm{e}^{\prime}\right)=\mathrm{e}^{\prime}$ and $\tau\left(\mathrm{e}^{\prime}\right)=-\mathrm{e}^{\prime}$.

Proof. Let $\beta$ be a path from $\Omega$ to $\mathrm{v}_{1}$ and set $\mathrm{e}^{\prime}=e^{\operatorname{ad}_{\beta}}(\mathrm{e})$. From Proposition 1.2, we get

$$
\begin{aligned}
d_{\Omega} \mathrm{e}^{\prime} & =d_{\Omega} e^{\operatorname{ad}_{\beta}}(\mathrm{e})=e^{\operatorname{ad}_{\beta}}\left(d_{\mathrm{v}_{1}} \mathrm{e}\right) \\
& =\beta * \mathrm{x}_{1} * \mathrm{x}_{2} * \mathrm{x}_{3} *(-\beta) .
\end{aligned}
$$

Let us observe that $\sigma(\beta) *\left(-\mathrm{x}_{1}\right)$ and $\beta$ are two paths from $\Omega$ to $\mathrm{v}_{1}$. From Lemma 2.2(ii) applied to $(-\beta) * \sigma(\beta) *\left(-\mathbf{x}_{1}\right)$, there exists $\lambda \in \mathbb{Q}$ such that

$$
\sigma(\beta)=\beta * \lambda\left(\mathrm{x}_{1} * \mathrm{x}_{2} * \mathrm{x}_{3}\right) * \mathrm{x}_{1} .
$$

Replacing $\sigma(\beta)$ by its value in $\sigma\left(d_{\Omega} \mathrm{e}^{\prime}\right)$, we get

$$
\begin{aligned}
\sigma d_{\Omega}\left(\mathrm{e}^{\prime}\right) & =\sigma(\beta) * \mathrm{x}_{2} * \mathrm{x}_{3} * \mathrm{x}_{1} *(-\sigma(\beta)) \\
& =\beta * \lambda\left(\mathrm{x}_{1} * \mathrm{x}_{2} * \mathrm{x}_{3}\right) * \mathrm{x}_{1} * \mathrm{x}_{2} * \mathrm{x}_{3} * \mathrm{x}_{1} *\left(-\mathrm{x}_{1}\right) *(-\lambda)\left(\mathrm{x}_{1} * \mathrm{x}_{2} * \mathrm{x}_{3}\right) *(-\beta) \\
& =\beta * \mathrm{x}_{1} * \mathrm{x}_{2} * \mathrm{x}_{3} *(-\beta) \\
& =d_{\Omega}\left(\mathrm{e}^{\prime}\right)
\end{aligned}
$$


On the other hand, let us recall $\tau\left(\mathrm{x}_{2}\right)=-\mathrm{x}_{2}, \tau\left(\mathrm{x}_{1}\right)=-\mathrm{x}_{3}, \tau\left(\mathrm{v}_{1}\right)=\mathrm{v}_{1}$ and $\tau\left(\mathrm{v}_{2}\right)=\mathrm{v}_{3}$. Applying Lemma 2.2(ii) to $(-\beta) * \tau(\beta)$, we obtain $\mu \in \mathbb{Q}$ such that

$$
\tau(\beta)=\beta * \mu\left(\mathrm{x}_{1} * \mathrm{x}_{2} * \mathrm{x}_{3}\right) .
$$

Once again, a similar computation gives

$$
\begin{aligned}
\tau\left(d_{\Omega}\left(\mathrm{e}^{\prime}\right)\right) & =\beta * \mu\left(\mathrm{x}_{1} * \mathrm{x}_{2} * \mathrm{x}_{3}\right) *\left(-\mathrm{x}_{3}\right) *\left(-\mathrm{x}_{2}\right) *\left(-\mathrm{x}_{1}\right) *(-\mu)\left(\mathrm{x}_{1} * \mathrm{x}_{2} * \mathrm{x}_{3}\right) *(-\beta) \\
& =\beta *\left(-\left(\mathrm{x}_{1} * \mathrm{x}_{2} * \mathrm{x}_{3}\right)\right) *(-\beta)=-d_{\Omega}\left(\mathrm{e}^{\prime}\right) .
\end{aligned}
$$

Problem. In Theorem 2.4, we prove that the Lie model of a graph consisting of a circuit contains a MC-element invariant along the group of automorphisms of the graph. Is this result true in general: does the Lie model of any finite graph have a MC-element invariant along the automorphisms of the graph?

\section{REFERENCES}

[1] Urtzi Buijs, Yves Félix, Aniceto Murillo, and Daniel Tanré, Lie models of simplicial sets and representability of the Quillen functor, ArXiv 1508.01442 (2015).

[2] — The Deligne groupoid of the Lawrence-Sullivan interval, Topology Appl. 204 (2016), 1-7. MR 3482697

[3] _ Maurer-Cartan elements in the Lie models of finite simplicial complexes, Canad. Math. Bull. 60 (2017), no. 3, 470-477. MR 3679722

[4] H. Bursztyn, V. Dolgushev, and S. Waldmann, Morita equivalence and characteristic classes of star products, J. Reine Angew. Math. 662 (2012), 95-163. MR 2876262

[5] N. Gadish, I. Griniasty, and R. Lawrence, An explicit symmetric DGLA model of a bi-gon, ArXiv 1705.08483 (2017).

[6] Ruth Lawrence and Dennis Sullivan, A formula for topology/deformations and its significance, Fund. Math. 225 (2014), no. 1, 229-242. MR 3205571

[7] Paul-Eugène Parent and Daniel Tanré, Lawrence-Sullivan models for the interval, Topology Appl. 159 (2012), no. 1, 371-378. MR 2852982 (2012j:55014)

[8] Daniel G. Quillen, Rational homotopy theory, Ann. of Math. (2) 90 (1969), 205-295. MR 0258031 (41 \#2678)

[9] Jean-Pierre Serre, Lie algebras and Lie groups, Lectures given at Harvard University, vol. 1964, W. A. Benjamin, Inc., New York-Amsterdam, 1965. MR 0218496

[10] Dennis Sullivan, Infinitesimal computations in topology, Inst. Hautes Études Sci. Publ. Math. (1977), no. 47, 269-331 (1978). MR 0646078 (58 \#31119)

Departamento de Álgebra, Geometría y Topología, Universidad de Málaga, Ap. 59, 29080-MÁlaga, EsPaÑa

E-mail address: ubuijs@uma.es

Institut de Mathématiques et Physique, Université Catholique de Louvain-la-Neuve, Louvain-LA-Neuve, Belgique

E-mail address: Yves.felix@uclouvain.be

Departamento de Álgebra, Geometría y Topología, Universidad de Málaga, Ap. 59, 29080-MÁlagA, EsPaÑA

E-mail address: aniceto@uma.es

Département de Mathématiques, UMR 8524, Université de Lille 1, 59655 Villeneuve D'Ascq Cedex, France

E-mail address: Daniel.Tanre@univ-lille1.fr 